\title{
UNIQUENESS OF NONLINEAR DIFFERENTIAL POLYNOMIALS SHARING SIMPLE AND DOUBLE 1-POINTS
}

INDRAJIT LAHIRI AND NINTU MANDAL

Received 19 October 2004 and in revised form 18 March 2005

We prove two theorems on the uniqueness of nonlinear differential polynomials, one of which improves a result of Fang and Hong.

\section{Introduction, definitions, and results}

Let $f$ and $g$ be two nonconstant meromorphic functions defined in the open complex plane $\mathbb{C}$. Let $k$ be a positive integer or infinity and $a \in \mathbb{C} \cup\{\infty\}$. We denote by $E_{k}(a ; f)$ the set of all $a$-points of $f$ with multiplicities not exceeding $k$, where an $a$-point is counted according to its multiplicity. If for some $a \in \mathbb{C} \cup\{\infty\}, E_{\infty)}(a ; f)=E_{\infty}(a ; g)$, we say that $f, g$ share the value $a \mathrm{CM}$ (counting multiplicities).

During the last few years, a considerable amount of work is being done on the uniqueness problem concerning differential polynomials (cf. $[1,3,5,8]$ ). Recently, Fang and Hong [1] proved the following result.

Theorem 1.1 [1]. Let $f$ and $g$ be two transcendental entire functions and let $n(\geq 11)$ be an integer. If $f^{n}(f-1) f^{\prime}$ and $g^{n}(g-1) g^{\prime}$ share $1 C M$, then $f \equiv g$.

In the paper, we prove the following two theorems, the first of which improves Theorem 1.1.

THeORem 1.2. Let $f$ and $g$ be two transcendental entire functions and let $n(\geq 10)$ be an integer. If $E_{2)}\left(1 ; f^{n}(f-1) f^{\prime}\right)=E_{2)}\left(1 ; g^{n}(g-1) g^{\prime}\right)$, then $f \equiv g$.

THeOREM 1.3. Let $f$ and $g$ be two transcendental meromorphic functions such that $\Theta(\infty ; f)$ $+\Theta(\infty ; g)>4 /(n+1)$ and let $n(\geq 17)$ be an integer. If $\left.E_{2}\right)\left(1 ; f^{n}(f-1) f^{\prime}\right)=E_{2}\left(1 ; g^{n}(g-\right.$ 1) $\left.g^{\prime}\right)$, then $f \equiv g$.

The following example shows that the condition $\Theta(\infty ; f)+\Theta(\infty ; g)>4 /(n+1)$ is sharp for Theorem 1.3.

Example 1.4. Let

$$
f=\frac{(n+2)\left(1-h^{n+1}\right)}{(n+1)\left(1-h^{n+2}\right)}, \quad g=\frac{(n+2) h\left(1-h^{n+1}\right)}{(n+1)\left(1-h^{n+2}\right)}, \quad h=\frac{\alpha^{2}\left(e^{z}-1\right)}{e^{z}-\alpha},
$$

where $\alpha=\exp (2 \pi i /(n+2))$ and $n$ is a positive integer. 
Then, $T(r, f)=(n+1) T(r, h)+O(1)$ and $T(r, g)=(n+1) T(r, h)+O(1)$. Further, we see that $h \neq \alpha, \alpha^{2}$ and a root of $h=1$ is not a pole of $f$ and $g$. Hence, $\Theta(\infty ; f)=\Theta(\infty ; g)=$ $2 /(n+1)$. Also $f^{n+1}(f /(n+2)-1 /(n+1)) \equiv g^{n+1}(g /(n+2)-1 /(n+1))$ and $f^{n}(f-1) f^{\prime} \equiv$ $g^{n}(g-1) g^{\prime}$ but $f \not \equiv g$.

Though we do not explain the standard notations of the value distribution theory (see [2]), we give the following definitions.

Definition 1.5 [4]. For $a \in \mathbb{C} \cup\{\infty\}$, denote by $N(r, a ; f \mid=1)$ the counting functions of simple $a$-points of $f$.

For a positive integer $m$, denote by $N(r, a ; f \mid \leq m)(N(r, a ; f \mid \geq m))$ the counting function of those $a$-points of $f$ whose multiplicities are not greater (less) than $m$, where each $a$-point is counted according to its multiplicity.

$\bar{N}(r, a ; f \mid \leq m)$ and $\bar{N}(r, a ; f \mid \geq m)$ are defined similarly, where in counting the $a$ points of $f$, the multiplicities are ignored.

Also $N(r, a ; f \mid<m), N(r, a ; f \mid>m), \bar{N}(r, a ; f \mid<m)$ and $\bar{N}(r, a ; f \mid>m)$ are defined analogously.

Definition $1.6[12]$. For $a \in \mathbb{C} \cup\{\infty\}$, put

$$
N_{k}(r, a ; f)=\bar{N}(r, a ; f)+\bar{N}(r, a ; f \mid \geq 2)+\bar{N}(r, a ; f \mid \geq 3)+\cdots+\bar{N}(r, a ; f \mid \geq k),
$$

where $k$ is a positive integer.

For a meromorphic function $f$, we denote by $S(r, f)$ any function satisfying $S(r, f)$ / $T(r, f) \rightarrow 0$ as $r \rightarrow \infty$ possibly outside a set of finite linear measure.

\section{Lemmas}

In this section, we present some lemmas which are needed in the sequel. We denote by $h$ the function

$$
h=\left(\frac{f^{\prime \prime}}{f^{\prime}}-\frac{2 f^{\prime}}{f-1}\right)-\left(\frac{g^{\prime \prime}}{g^{\prime}}-\frac{2 g^{\prime}}{g-1}\right) .
$$

Lemma 2.1. If $E_{1)}(1 ; f)=E_{1)}(1 ; g)$ and $h \not \equiv 0$, then

$$
N(r, 1 ; f \mid \leq 1)=N(r, 1 ; g \mid \leq 1) \leq N(r, 0 ; h) \leq N(r, \infty ; h)+S(r, f)+S(r, g) .
$$

Proof. Since the functions $f$ and $g$ have the same simple one-points, there exists a meromorphic function $\alpha$ such that $\alpha \neq 0$ when $f-1$ has a simple zero and $\alpha$ has no simple zero where $f \neq 1$ and $g=\alpha(f-1)+1$. It is now easy to verify by direct computation that the function $h$ is zero whenever $f-1$ has a simple zero. This proves the lemma.

LemmA 2.2. If $E_{2)}(1 ; f)=E_{2)}(1 ; g)$ and $h \neq \equiv 0$, then

$$
\begin{aligned}
N(r, \infty ; h) \leq & \bar{N}(r, \infty ; f \mid \geq 2)+\bar{N}(r, 0 ; f \mid \geq 2)+\bar{N}(r, \infty ; g \mid \geq 2) \\
& +\bar{N}(r, 0 ; g \mid \geq 2)+\bar{N}(r, 1 ; f \mid \geq 3)+\bar{N}(r, 1 ; g \mid \geq 3) \\
& +\bar{N}_{0}\left(r, 0 ; f^{\prime}\right)+\bar{N}_{0}\left(r, 0 ; g^{\prime}\right),
\end{aligned}
$$


where $\bar{N}_{0}\left(r, 0 ; f^{\prime}\right)$ and $\bar{N}_{0}\left(r, 0 ; g^{\prime}\right)$ are the reduced counting functions of the zeros of $f^{\prime}$ and $g^{\prime}$ which are not the zeros of $f(f-1)$ and $g(g-1)$, respectively.

Proof. We can easily verify that possible poles of $h$ occur at (i) multiple zeros of $f, g$; (ii) multiple poles of $f, g$; (iii) zeros of $f-1, g-1$ with multiplicities greater than or equal to 3; (iv) zeros of $f^{\prime}$ which are not the zeros of $f(f-1)$; (v) zeros of $g^{\prime}$ which are not the zeros of $g(g-1)$.

Since all the poles of $h$ are simple, the lemma follows from above. This proves the lemma.

Lemma 2.3 [6]. If $N\left(r, 0 ; f^{(k)} \mid f \neq 0\right)$ denotes the counting function of those zeros of $f^{(k)}$ which are not the zeros of $f$, where a zero of $f^{(k)}$ is counted according to its multiplicity, then

$$
N\left(r, 0 ; f^{(k)} \mid f \neq 0\right) \leq k \bar{N}(r, \infty ; f)+N(r, 0 ; f \mid<k)+k \bar{N}(r, 0 ; f \mid \geq k)+S(r, f) .
$$

Lemma 2.4. If $E_{2)}(1 ; f)=E_{2)}(1 ; g)$ and $h \not \equiv 0$, then

$$
\begin{aligned}
T(r, f)+T(r, g) \leq & \{3 \bar{N}(r, 0 ; f)+2 \bar{N}(r, 0 ; f \mid \geq 2)\}+\{3 \bar{N}(r, \infty ; f)+2 \bar{N}(r, \infty ; f \mid \geq 2)\} \\
& +\{3 \bar{N}(r, 0 ; g)+2 \bar{N}(r, 0 ; g \mid \geq 2)\}+\{3 \bar{N}(r, \infty ; g)+2 \bar{N}(r, \infty ; g \mid \geq 2)\} \\
& +S(r, f)+S(r, g) .
\end{aligned}
$$

Proof. By Nevanlinna's second fundamental theorem and Lemmas 2.1 and 2.2, we get

$$
\begin{aligned}
T(r, f) \leq & \bar{N}(r, 0 ; f)+\bar{N}(r, \infty ; f)+\bar{N}(r, 1 ; f)-N_{0}\left(r, 0 ; f^{\prime}\right)+S(r, f) \\
\leq & \bar{N}(r, 0 ; f)+\bar{N}(r, \infty ; f)+\bar{N}(r, 1 ; f \mid \geq 2)+\bar{N}(r, 0 ; f \mid \geq 0) \\
& +\bar{N}(r, \infty ; f \mid \geq 2)+\bar{N}(r, 0 ; g \mid \geq 2)+\bar{N}(r, 1 ; f \mid \geq 3) \\
& +\bar{N}(r, 1 ; g \mid \geq 3)+\bar{N}_{0}\left(r, 0 ; g^{\prime}\right)+S(r, f)+S(r, g) .
\end{aligned}
$$

Also we get

$$
\begin{aligned}
& \bar{N}(r, 1 ; f \mid \geq 2)+\bar{N}(r, 1 ; f \mid \geq 3) \leq N\left(r, 0 ; f^{\prime} \mid f \neq 0\right), \\
& \bar{N}(r, 1 ; g \mid \geq 3)+\bar{N}_{0}\left(r, 0 ; g^{\prime}\right) \leq N\left(r, 0 ; g^{\prime} \mid g \neq 0\right) .
\end{aligned}
$$

So from (2.6), we get

$$
\begin{aligned}
T(r, f) \leq & \bar{N}(r, 0 ; f)+\bar{N}(r, 0 ; f \mid \geq 2)+\bar{N}(r, \infty ; f)+\bar{N}(r, \infty ; f \mid \geq 2) \\
& +\bar{N}(r, 0 ; g \mid \geq 2)+\bar{N}(r, \infty ; g \mid \geq 2)+N\left(r, 0 ; f^{\prime} \mid f \neq 0\right) \\
& +N\left(r, 0 ; g^{\prime} \mid g \neq 0\right)+S(r, f)+S(r, g) .
\end{aligned}
$$


1936 Uniqueness of nonlinear differential polynomials

Similarly,

$$
\begin{aligned}
T(r, g) \leq & \bar{N}(r, 0 ; g)+\bar{N}(r, 0 ; g \mid \geq 2)+\bar{N}(r, \infty ; g)+\bar{N}(r, \infty ; g \mid \geq 2) \\
& +\bar{N}(r, 0 ; f \mid \geq 2)+\bar{N}(r, \infty ; f \mid \geq 2)+N\left(r, 0 ; f^{\prime} \mid f \neq 0\right) \\
& +N\left(r, 0 ; g^{\prime} \mid g \neq 0\right)+S(r, f)+S(r, g) .
\end{aligned}
$$

Adding (2.8) and (2.9) and using Lemma 2.3, we obtain the following lemma.

LEMMA 2.5 [10]. Let $f$ be a nonconstant meromorphic function and $P(f)=a_{0}+a_{1} f+$ $a_{2} f^{2}+\cdots+a_{n} f^{n}$, where $a_{0}, a_{1}, \ldots, a_{n}$ are constants and $a_{n} \neq 0$. Then

$$
T(r, P(f))=n T(r, f)+O(1)
$$

Lemma 2.6. Let $f$ and $g$ be two nonconstant meromorphic functions such that

$$
\Theta(\infty ; f)+\Theta(\infty ; g)>\frac{4}{n+1}
$$

where $n(\geq 2)$ is an integer. Then

$$
f^{n+1}(a f+b) \equiv g^{n+1}(a g+b)
$$

implies that $f \equiv g$, where $a, b$ are finite nonzero constants.

Proof. Let

$$
f^{n+1}(a f+b) \equiv g^{n+1}(a g+b)
$$

and $f \not \equiv g$. We consider the following two cases.

Case 1. Let $y=g / f$ be a constant. Then $y \neq 1$ and from (2.13), we get

$$
a f\left(1-y^{n+2}\right) \equiv-b\left(1-y^{n+1}\right)
$$

from which it follows that $y^{n+1} \neq 1, y^{n+2} \neq 1$, and

$$
f \equiv-\frac{b\left(1-y^{n+1}\right)}{a\left(1-y^{n+2}\right)}
$$

This is a contradiction because $f$ is nonconstant.

Case 2. Let $y=g / f$ be not a constant. Then from (2.13), we get

$$
f \equiv \frac{b}{a}\left(\frac{y^{n+1}}{1+y+y^{2}+\cdots+y^{n+1}}-1\right) .
$$


From (2.16), we obtain by Nevanlinna's first fundamental theorem and Lemma 2.5

$$
\begin{aligned}
T(r, f) & =T\left(r, \sum_{j=0}^{n+1} \frac{1}{y^{j}}\right)+S(r, y) \\
& =(n+1) T\left(r, \frac{1}{y}\right)+S(r, y) \\
& =(n+1) T(r, y)+S(r, y) .
\end{aligned}
$$

Now we note that a pole of $y$ is not a pole of $(b / a)\left(y^{n+1} /\left(1+y+y^{2}+\cdots+y^{n+1}\right)-1\right)$. So from (2.16), we get

$$
\sum_{k=1}^{n+1} \bar{N}\left(r, u_{k} ; y\right) \leq \bar{N}(r, \infty ; f),
$$

where $u_{k}=\exp (2 k \pi i /(n+2))$ for $k=1,2, \ldots, n+1$.

So by Nevanlinna's second fundamental theorem, we obtain

$$
\begin{aligned}
(n-1) T(r, y) & \leq \sum_{k=1}^{n+1} \bar{N}\left(r, u_{k} ; y\right)+S(r, y) \\
& \leq \bar{N}(r, \infty ; f)+S(r, y) \\
& <(1-\Theta(\infty ; f)+\varepsilon) T(r, f)+S(r, y) \\
& =(n+1)(1-\Theta(\infty ; f)+\varepsilon) T(r, y)+S(r, y),
\end{aligned}
$$

where $\varepsilon(>0)$.

Again putting $y_{1}=1 / y$, noting that $T(r, y)=T\left(r, y_{1}\right)+O(1)$, and proceeding as above we get

$$
(n-1) T(r, y) \leq(n+1)(1-\Theta(\infty ; g)+\varepsilon) T(r, y)+S(r, y)
$$

Since $\Theta(\infty ; f)+\Theta(\infty ; g)>4 /(n+1)$, there exists a $\delta(>0)$ such that $\Theta(\infty ; f)+\Theta(\infty ; g)>$ $\delta+4 /(n+1)$. Now adding (2.19) and (2.20), we obtain

$$
\begin{aligned}
2(n-1) T(r, y) & \leq(n+1)(2-\Theta(\infty ; f)-\Theta(\infty ; g)+2 \varepsilon) T(r, y)+S(r, y) \\
& \leq(n+1)\left(2-\frac{4}{n+1}-\delta+2 \varepsilon\right) T(r, y)+S(r, y),
\end{aligned}
$$

and so $(\delta-2 \varepsilon) T(r, y) \leq S(r, y)$, which is a contradiction for any $\varepsilon(0<2 \varepsilon<\delta)$. Therefore, $f \equiv g$ and the proof of the lemma is complete.

LEMma 2.7. Let $f$ and $g$ be nonconstant meromorphic functions. Then

$$
f^{n}(f-1) f^{\prime} g^{n}(g-1) g^{\prime} \not \equiv 1,
$$

where $n(\geq 5)$ is an integer. 
1938 Uniqueness of nonlinear differential polynomials

Proof. Let

$$
f^{n}(f-1) f^{\prime} g^{n}(g-1) g^{\prime} \equiv 1 .
$$

Let $z_{0}$ be a 1 -point of $f$ with multiplicity $p(\geq 1)$. Then $z_{0}$ is a pole of $g$ with multiplicity $q(\geq 1)$ such that

$$
2 p-1=(n+2) q+1
$$

that is,

$$
2 p=(n+2) q+2 \geq n+4
$$

that is,

$$
p \geq \frac{n+4}{2}
$$

Let $z_{0}$ be a zero of $f$ with multiplicity $p(\geq 1)$ and let it be a pole of $g$ with multiplicity $q(\geq 1)$. Then

$$
(n+1) p-1=(n+2) q+1 .
$$

From (2.27), we get

$$
q+2=(n+1)(p-q) \geq n+1,
$$

that is,

$$
q \geq n-1
$$

Again from (2.27), we get

$$
(n+1) p=(n+2) q+2 \geq(n+2)(n-1)+2,
$$

that is,

$$
p \geq \frac{(n+2)(n-1)+2}{n+1}=n .
$$

Since a pole of $f$ is either a zero of $g(g-1)$ or a zero of $g^{\prime}$, we see that

$$
\begin{aligned}
\bar{N}(r, \infty ; f) & \leq \bar{N}(r, 0 ; g)+\bar{N}(r, 1 ; g)+\bar{N}_{0}\left(r, 0 ; g^{\prime}\right) \\
& \leq \frac{1}{n} N(r, 0 ; g)+\frac{2}{n+4} N(r, 1 ; g)+\bar{N}_{0}\left(r, 0 ; g^{\prime}\right) \\
& \leq\left(\frac{1}{n}+\frac{2}{n+4}\right) T(r, g)+\bar{N}_{0}\left(r, 0 ; g^{\prime}\right) .
\end{aligned}
$$

Now by Nevanlinna's second fundamental theorem, we obtain

$$
\begin{aligned}
T(r, f) & \leq \bar{N}(r, 0 ; f)+\bar{N}(r, 1 ; f)+\bar{N}(r, \infty ; f)-\bar{N}_{0}\left(r, 0 ; f^{\prime}\right)+S(r, f) \\
& \leq \frac{1}{n} N(r, 0 ; f)+\frac{2}{n+4} N(r, 1 ; f)+\bar{N}(r, \infty ; f)-\bar{N}_{0}\left(r, 0 ; f^{\prime}\right)+S(r, f),
\end{aligned}
$$


that is,

$$
\left(1-\frac{1}{n}-\frac{2}{n+4}\right) T(r, f) \leq\left(\frac{1}{n}+\frac{2}{n+4}\right) T(r, g)+\bar{N}_{0}\left(r, 0 ; g^{\prime}\right)-\bar{N}_{0}\left(r, 0 ; f^{\prime}\right)+S(r, f) .
$$

Similarly, we get

$$
\left(1-\frac{1}{n}-\frac{2}{n+4}\right) T(r, g) \leq\left(\frac{1}{n}+\frac{2}{n+4}\right) T(r, f)+\bar{N}_{0}\left(r, 0 ; f^{\prime}\right)-\bar{N}_{0}\left(r, 0 ; g^{\prime}\right)+S(r, g) \text {. }
$$

Adding (2.34) and (2.35), we get

$$
\left(1-\frac{2}{n}-\frac{4}{n+4}\right)\{T(r, f)+T(r, g)\} \leq S(r, f)+S(r, g)
$$

which is a contradiction because $1-(2 / n)-4 /(n+4)>0$. This proves the lemma.

LEMma 2.8. Let $f$ and $g$ be two nonconstant meromorphic functions and

$$
F=f^{n+1}\left(\frac{f}{n+2}-\frac{1}{n+1}\right), \quad G=g^{n+1}\left(\frac{g}{n+2}-\frac{1}{n+1}\right)
$$

where $n(\geq 4)$ is an integer. Then $F^{\prime} \equiv G^{\prime}$ implies that $F \equiv G$.

Proof. If $F^{\prime} \equiv G^{\prime}$, then $F \equiv G+c$, where $c$ is a constant. Let $c \neq 0$. Then by Nevanlinna's second fundamental theorem and Lemma 2.5, we get

$$
\begin{aligned}
(n+2) T(r, f) \leq & \bar{N}(r, \infty ; F)+\bar{N}(r, 0 ; F)+\bar{N}(r, c ; F)+S(r, F) \\
= & \bar{N}(r, \infty ; f)+\bar{N}(r, 0 ; f)+\bar{N}\left(r, \frac{n+2}{n+1} ; f\right)+\bar{N}(r, 0 ; g) \\
& +\bar{N}\left(r, \frac{n+2}{n+1} ; g\right)+S(r, f) \leq 3 T(r, f)+2 T(r, g)+S(r, f),
\end{aligned}
$$

that is,

$$
(n-1) T(r, f) \leq 2 T(r, g)+S(r, f) .
$$

Similarly, we get

$$
(n-1) T(r, g) \leq 2 T(r, f)+S(r, g) \text {. }
$$

This shows that

$$
(n-3) T(r, f)+(n-3) T(r, g) \leq S(r, f)+S(r, g),
$$

which is a contradiction. Therefore $c=0$ and so $F \equiv G$. This proves the lemma. 
1940 Uniqueness of nonlinear differential polynomials

LEMmA 2.9. If $F$ and $G$ are defined as in Lemma 2.8, then

(i) $T(r, F) \leq T\left(r, F^{\prime}\right)+N(r, 0 ; f)+N(r,(n+2) /(n+1) ; f)-N(r, 1 ; f)-N\left(r, 0 ; f^{\prime}\right)$ $+S(r, f)$,

(ii) $T(r, G) \leq T\left(r, G^{\prime}\right)+N(r, 0 ; g)+N(r,(n+2) /(n+1) ; g)-N(r, 1 ; g)-N\left(r, 0 ; g^{\prime}\right)$ $+S(r, g)$.

Proof. We prove (i) because (ii) is similar. Now in view of Nevanlinna's first fundamental theorem and Lemma 2.5, we get

$$
\begin{aligned}
T(r, F)= & T\left(r, \frac{1}{F}\right)+O(1) \\
= & N(r, 0 ; F)+m\left(r, \frac{1}{F}\right)+O(1) \leq N(r, 0 ; F)+m\left(r, \frac{F^{\prime}}{F}\right)+m\left(r, \frac{1}{F^{\prime}}\right) \\
= & T\left(r, F^{\prime}\right)+N(r, 0 ; F)-N\left(r, 0 ; F^{\prime}\right)+S(r, F) \\
= & T\left(r, F^{\prime}\right)+(n+1) N(r, 0 ; f)+N\left(r, \frac{n+2}{n+1} ; f\right)-n N(r, 0 ; f) \\
& -N(r, 1 ; f)-N\left(r, 0 ; f^{\prime}\right)+S(r, f) \\
= & T\left(r, F^{\prime}\right)+N(r, 0 ; f)+N\left(r, \frac{n+2}{n+1} ; f\right)-N(r, 1 ; f)-N\left(r, 0 ; f^{\prime}\right)+S(r, f) .
\end{aligned}
$$

This proves the lemma.

Lemma 2.10 [7]. Let $f$ be a nonconstant meromorphic function and let $k$ be a positive integer. Then

$$
N_{2}\left(r, 0 ; f^{(k)}\right) \leq k \bar{N}(r, \infty ; f)+N_{2+k}(r, 0 ; f)+S(r, f)
$$

Lemma 2.11 [13]. If $h \equiv 0$ then $f$, $g$ share $1 C M$.

Proof. Since $h \equiv 0$, integrating, we get $f^{\prime} /(f-1)^{2} \equiv A g^{\prime} /(g-1)^{2}$, where $A$ is a nonzero constant. From this, the lemma follows.

LEMMA $2.12[9,11]$. If $f$ and $g$ share $1 C M$, then one of the following cases holds:

(i) $T(r, f)+T(r, g) \leq 2\left\{N_{2}(r, 0 ; f)+N_{2}(r, 0 ; g)+N_{2}(r, \infty ; f)+N_{2}(r, \infty ; g)\right\}+S(r, f)+$ $S(r, g)$;

(ii) $f \equiv g$;

(iii) $f g \equiv 1$.

\section{Proof of theorems}

We prove Theorem 1.3 only because Theorem 1.2 can be proved similarly noting that in this case, $\bar{N}(r, \infty ; f) \equiv \bar{N}(r, \infty ; g) \equiv 0$.

Proof of Theorem 1.3. Let $F$ and $G$ be defined as in Lemma 2.8 and $F_{1}=F^{\prime}=f^{n}(f-1) f^{\prime}$, $G_{1}=G^{\prime}=g^{n}(g-1) g^{\prime}$. Also we put

$$
H=\left(\frac{F_{1}^{\prime \prime}}{F_{1}^{\prime}}-\frac{2 F_{1}^{\prime}}{F_{1}-1}\right)-\left(\frac{G_{1}^{\prime \prime}}{G_{1}^{\prime}}-\frac{2 G_{1}^{\prime}}{G_{1}-1}\right) .
$$


If possible, let $H \not \equiv 0$. Then by Lemmas 2.4 and 2.9, we get

$$
\begin{aligned}
T(r, F)+T(r, G) \leq & T\left(r, F^{\prime}\right)+T\left(r, G^{\prime}\right)+N(r, 0 ; f)+N\left(r, \frac{n+2}{n+1} ; f\right) \\
& -N(r, 1 ; f)-N\left(r, 0 ; f^{\prime}\right)+N(r, 0 ; g)+N\left(r, \frac{n+2}{n+1} ; g\right) \\
& -N(r, 1 ; g)-N\left(r, 0 ; g^{\prime}\right)+S(r, f)+S(r, g) \\
\leq & \left\{3 \bar{N}\left(r, 0 ; f^{n}(f-1) f^{\prime}\right)+2 \bar{N}\left(r, 0 ; f^{n}(f-1) f^{\prime} \mid \geq 2\right)\right\} \\
& +\left\{3 \bar{N}\left(r, 0 ; g^{n}(g-1) g^{\prime}\right)+2 \bar{N}\left(r, 0 ; g^{n}(g-1) g^{\prime} \mid \geq 2\right)\right\} \\
& +5 \bar{N}(r, \infty ; f)+5 \bar{N}(r, \infty ; g)+N(r, 0 ; f)+N\left(r, \frac{n+2}{n+1} ; f\right) \\
& -N(r, 1 ; f)-N\left(r, 0 ; f^{\prime}\right)+N(r, 0 ; g)+N\left(r, \frac{n+2}{n+1} ; g\right) \\
& -N(r, 1 ; g)-N\left(r, 0 ; g^{\prime}\right)+S(r, f)+S(r, g) \\
\leq & 6 N(r, 0 ; f)+2 N_{2}(r, 1 ; f)+2 N_{2}\left(r, 0 ; f^{\prime}\right)+5 \bar{N}(r, \infty ; f) \\
& +N\left(r, \frac{n+2}{n+1} ; f\right)+6 N(r, 0 ; g)+2 N_{2}(r, 1 ; g)+2 N_{2}\left(r, 0 ; g^{\prime}\right) \\
& +5 \bar{N}(r, \infty ; g)+N\left(r, \frac{n+2}{n+1} ; g\right)+S(r, f)+S(r, g) .
\end{aligned}
$$

So by Lemmas 2.5 and 2.10, we get

$$
\begin{aligned}
(n+2) T(r, f)+(n+2) T(r, g) \leq & 9 T(r, f)+7 \bar{N}(r, \infty ; f)+2 N_{3}(r, 0 ; f)+9 T(r, g) \\
& +7 \bar{N}(r, \infty ; g)+2 N_{3}(r, 0 ; g)+S(r, f)+S(r, g) \\
\leq & 18 T(r, f)+18 T(r, g)+S(r, f)+S(r, g),
\end{aligned}
$$

that is,

$$
(n-16) T(r, f)+(n-16) T(r, g) \leq S(r, f)+S(r, g)
$$

which is a contradiction.

Therefore $H \equiv 0$ and so by Lemma $2.11, F_{1}$ and $G_{1}$ share 1 CM. In a similar manner as above, we can verify that the following inequality does not hold:

$$
\begin{aligned}
T\left(r, F_{1}\right)+T\left(r, G_{1}\right) \leq & 2\left\{N_{2}\left(r, 0 ; F_{1}\right)+N_{2}\left(r, \infty ; F_{1}\right)+N_{2}\left(r, 0 ; G_{1}\right)+N_{2}\left(r, \infty ; G_{1}\right)\right\} \\
& +S\left(r, F_{1}\right)+S\left(r, G_{1}\right) .
\end{aligned}
$$

So by Lemmas $2.12,2.7,2.8$, and 2.6 , we get $f \equiv g$. This proves the theorem.

\section{Acknowledgment}

The authors are thankful to the referees for valuable suggestions towards the improvement of the paper. 


\section{Uniqueness of nonlinear differential polynomials}

\section{References}

[1] M.-L. Fang and W. Hong, A unicity theorem for entire functions concerning differential polynomials, Indian J. Pure Appl. Math. 32 (2001), no. 9, 1343-1348.

[2] W. K. Hayman, Meromorphic Functions, Oxford Mathematical Monographs, Clarendon Press, Oxford, 1964.

[3] I. Lahiri, Uniqueness of meromorphic functions when two linear differential polynomials share the same 1-points, Ann. Polon. Math. 71 (1999), no. 2, 113-128.

[4] , Value distribution of certain differential polynomials, Int. J. Math. Math. Sci. 28 (2001), no. 2, 83-91.

[5] Linear differential polynomials sharing the same 1-points with weight two, Ann. Polon. Math. 79 (2002), no. 2, 157-170.

[6] I. Lahiri and S. Dewan, Value distribution of the product of a meromorphic function and its derivative, Kodai Math. J. 26 (2003), no. 1, 95-100.

[7] I. Lahiri and A. Sarkar, Uniqueness of a meromorphic function and its derivative, JIPAM. J. Inequal. Pure Appl. Math. 5 (2004), no. 1, article 20.

[8] W. C. Lin, Uniqueness of differential polynomials and a problem of Lahiri, Pure Appl. Math. (Xi'an) 17 (2001), no. 2, 104-110 (Chinese).

[9] E. Mues and M. Reinders, Meromorphic functions sharing one value and unique range sets, Kodai Math. J. 18 (1995), no. 3, 515-522.

[10] C.-C. Yang, On deficiencies of differential polynomials. II, Math. Z. 125 (1972), 107-112.

[11] C.-C. Yang and X. H. Hua, Uniqueness and value-sharing of meromorphic functions, Ann. Acad. Sci. Fenn. Math. 22 (1997), no. 2, 395-406.

[12] H.-X. Yi, On characteristic function of a meromorphic function and its derivative, Indian J. Math. 33 (1991), no. 2, 119-133.

[13] Some further results on uniqueness of meromorphic functions, Complex Var. Theory Appl. 38 (1999), no. 4, 375-385.

Indrajit Lahiri: Department of Mathematics, University of Kalyani, Kalyani-741235, West Bengal, India

E-mail address: ilahiri@vsnl.com

Nintu Mandal: Department of Mathematics, University of Kalyani, Kalyani-741235, West Bengal India

E-mail address: nintu_mandal@yahoo.com 


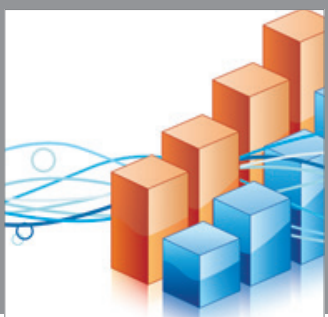

Advances in

Operations Research

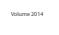

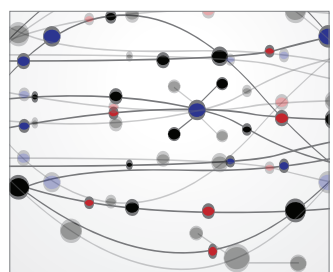

\section{The Scientific} World Journal
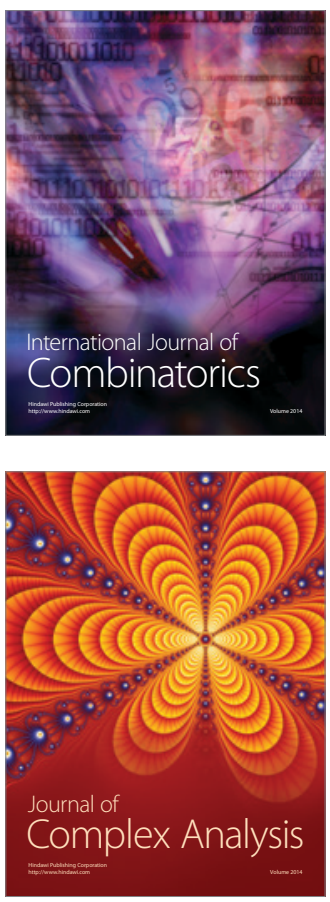

International Journal of

Mathematics and

Mathematical

Sciences
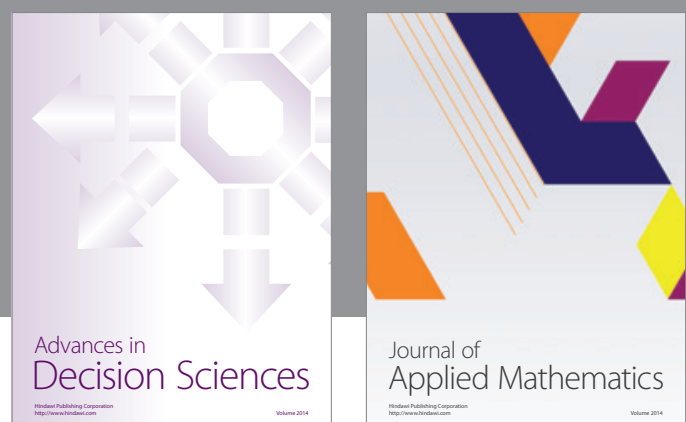

Journal of

Applied Mathematics
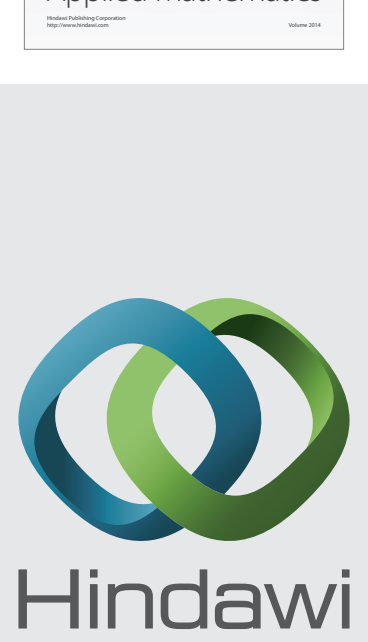

Submit your manuscripts at http://www.hindawi.com
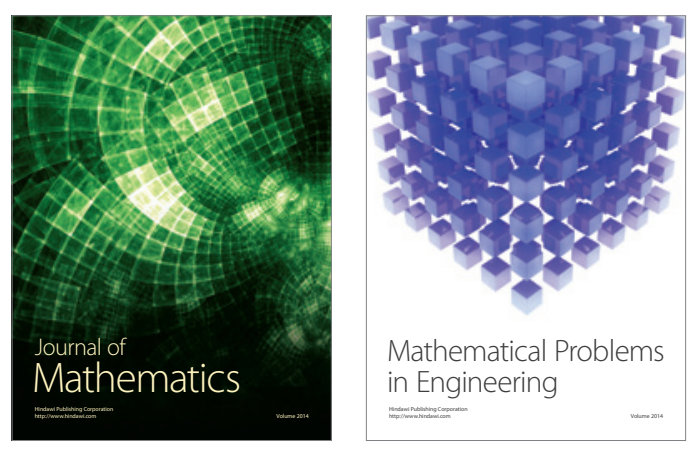

Mathematical Problems in Engineering
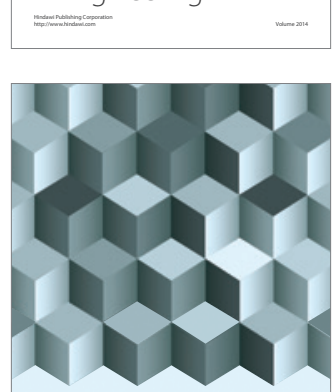

Journal of

Function Spaces
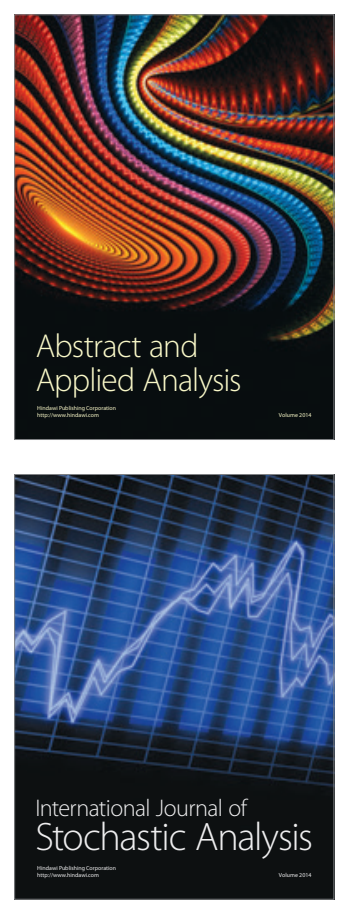

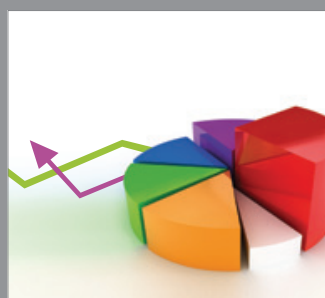

ournal of

Probability and Statistics

Promensencen
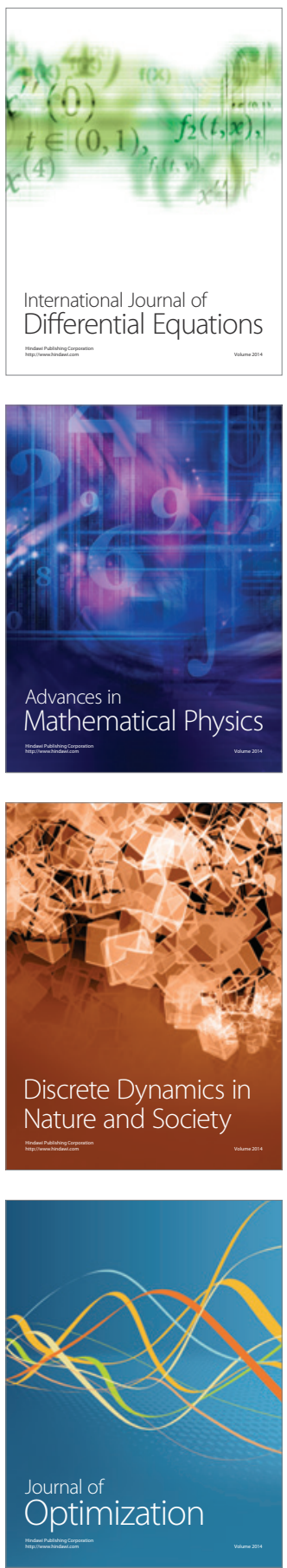